\title{
High risk of pulmonary embolism in acute respiratory distress syndrome related to COVID-19: an observational controlled-cohort study
}

\author{
Victor de Roubin ${ }^{1 \#}$, Faustine Reynaud ${ }^{1 \#}$, Rémi Coudroy ${ }^{1,2}$, Maeva Rodriguez $^{1}$, Grégoire Monseau ${ }^{1}$, \\ Florent Joly ${ }^{1}$, Justine Bardin ${ }^{1}$, Florence Boissier ${ }^{1}$, Delphine Chatellier ${ }^{1}$, Anne Veinstein ${ }^{1}$, René Robert ${ }^{1,2}$, \\ Jean-Pierre Frat ${ }^{1,2}$, Arnaud W. Thille ${ }^{1,2} \wedge$ \\ ${ }^{1}$ Centre Hospitalier Universitaire de Poitiers, Médecine Intensive Réanimation, Poitiers, France; ${ }^{2}$ INSERM CIC 1402 ALIVE Research Group, \\ University of Poitiers, Poitiers, France. \\ Contributions: (I) Conception and design: AW Thille; (II) Administrative support: AW Thille; (III) Provision of study materials or patients: All \\ authors; (IV) Collection and assembly of data: V de Roubin, F Reynaud, AW Thille; (V) Data analysis and interpretation: All authors; (VI) \\ Manuscript writing: All authors; (VII) Final approval of manuscript: All authors. \\ \#These authors contributed equally to this work. \\ Correspondence to: Pr. Arnaud W. Thille. Médecine Intensive Réanimation, CHU de Poitiers, 2 rue la Milétrie, 86021 Poitiers Cedex, France. \\ Email: aw.thille@gmail.com.
}

Background: COVID-19 may induce endovascular injury of pulmonary vessels and could be associated with increased risk of pulmonary embolism. The main objective was to compare the incidence of pulmonary embolism in patients with acute respiratory distress syndrome (ARDS) related to COVID-19 versus patients with pulmonary ARDS unrelated to COVID-19.

Methods: This is an observational controlled-cohort study performed at a single center of a university teaching hospital in France. The incidence of pulmonary embolism was prospectively assessed using computed tomography pulmonary angiography (CTPA) in patients with ARDS related to COVID-19 and compared to patients from a 3-year historical cohort of patients with pulmonary ARDS unrelated to COVID-19. In patients with ARDS related to COVID-19, CTPA was performed approximately 7 days after intubation or earlier in case of respiratory or hemodynamic worsening.

Results: CTPA was performed in 29 out of the 42 patients (69\%) with ARDS related to COVID-19 and in 51 out of the 156 patients (33\%) from the historical cohort of patients with pulmonary ARDS unrelated to COVID-19. Incidence of pulmonary embolism was $40 \%(17 / 42)$ in patients with ARDS related to COVID-19 and 3\% (5/156) in the historical cohort $(\mathrm{P}=0.001)$. The proportion of patients with pulmonary embolism among all patients who had CTPA was 59\% (17/29) in patients with ARDS related to COVID-19 and $10 \%(5 / 51)$ in the historical cohort $(\mathrm{P}=0.0001)$. After adjustment on the interval between ICU admission and computed tomography, COVID-19 remained independently associated with pulmonary embolism.

Conclusions: Pulmonary embolism was particularly frequent in patients with ARDS related to COVID-19, thereby suggesting that CTPA should be systematically performed in these patients.

Keywords: Acute respiratory distress syndrome (ARDS); COVID-19; pulmonary embolism; mechanical ventilation; computed tomography pulmonary angiography (CTPA)

Submitted Oct 08, 2020. Accepted for publication Jan 03, 2021.

doi: $10.21037 /$ atm-20-6796

View this article at: http://dx.doi.org/10.21037/atm-20-6796

$\wedge$ ORCID: 0000-0002-7798-6715. 


\section{Introduction}

COVID-19 due to severe acute respiratory syndrome coronavirus (SARS-CoV-2) may be complicated by acute respiratory distress syndrome (ARDS) with bilateral pulmonary infiltrates and profound hypoxemia requiring mechanical ventilation $(1,2)$. Diffuse alveolar damage characterized by hyaline membranes, interstitial edema, cell necrosis and proliferation, is the morphological hallmark picture of the lung in patients with ARDS (3). Unlike common causes of ARDS, COVID-19 may induce endovascular injury of pulmonary vessels and could be associated with an increased risk of pulmonary embolism. Several series of clinical autopsies have found abnormally high rates of pulmonary embolism in deceased patients with ARDS related to COVID-19 $(4,5)$. In a series of 11 clinical autopsies from Austria, pulmonary artery thrombosis was observed in all patients, in addition to usual diffuse alveolar damage (4). In another series of 12 clinical autopsies from Germany, pulmonary embolism was found to be the main reason of death in four patients (5).

Several clinical or radiological studies have also reported a high incidence of pulmonary embolism among patients with COVID-19 and respiratory failure, with a rate approximating $20 \%$ (6-10). However, computed tomography pulmonary angiography (CTPA) is not a routine exam in ARDS management, which is one reason why the actual incidence of pulmonary embolism in patients with ARDS has been inadequately studied.

This study aimed at determining whether the incidence of pulmonary embolism was actually higher in patients with ARDS related to COVID-19 than in patients with pulmonary ARDS unrelated to COVID-19.

We present the following article in accordance with the STROBE reporting checklist (available at http://dx.doi. org/10.21037/atm-20-6796).

\section{Methods}

\section{Design and patients}

This is a single-center observational controlled-cohort study including patients intubated with clinical criteria for ARDS (11).

Patients who did not require intubation and invasive mechanical ventilation were not included. In the studied group, all patients with ARDS related to COVID-19 (confirmed by positive RT-PCR) admitted to ICU between the $23^{\text {rd }}$ March (first patient admitted for ARDS related to COVID-19) and $4^{\text {th }}$ June 2020 (discharge date of the last patient) were included. In the control group, all medical charts were reviewed by two intensivists over a 3 -year period (FR and VDR) to identify all intubated patients who met the criteria for pulmonary ARDS, i.e., related to pneumonia, aspiration, inhalation or lung contusion, whereas patients with extra-pulmonary ARDS were excluded (11). As pathogenic pathways could be different between pulmonary and extra-pulmonary ARDS, we decided to include only homogeneous patients with pulmonary ARDS characterized by direct insult of alveolar epithelium, and not patients with extra-pulmonary ARDS characterized by insult of pulmonary vascular endothelium (12). For all patients, prophylactic anticoagulation was administered as daily subcutaneous 4,000 IU enoxaparin in the absence of contraindication. The study was conducted in accordance with the Declaration of Helsinki (as revised in 2013), and was approved by the local Ethics committee of Poitiers (registration number: CHU86RECH-R2020-05-09). Given its observational nature, informed consent was waived.

\section{Outcomes}

The primary outcome was the proportion of patients with pulmonary embolism diagnosed using CTPA while under invasive mechanical ventilation. CTPA performed before ICU admission or before intubation was not retained in the analysis. When established, pulmonary embolism was classified as troncular, lobar, segmental or sub-segmental, based on the location of the most proximal endoluminal defect. The secondary outcome involved variables associated with pulmonary embolism among all patients having undergone CTPA.

As the pandemic occurred later in our region than in other parts of Europe, we were aware of a potential high risk of pulmonary embolism in patients with ARDS related to COVID-19. Consequently, we planned in advance to systematically perform CTPA approximately 7 days after intubation or earlier in case of respiratory or hemodynamic worsening, our objective being to confirm or rule out pulmonary embolism in all patients. By contrast, CTPA was not systematic in the control group with pulmonary ARDS unrelated to COVID-19, and was performed only in patients who had suspected pulmonary embolism or infectious pulmonary complication (pulmonary abscess or pleural empyema). 


\section{Statistical analysis}

Continuous variables were given in mean \pm standard deviation or median $\left[25^{\text {th }}-75^{\text {th }}\right.$ percentiles $]$ and compared using the Student's $t$-test or the Mann-Whitney test as appropriate. Categorical variables were given in number and percentage and compared by means of the $\chi^{2}$ test or the Fischer's exact test as appropriate.

Patients with ARDS related to COVID-19 were compared to those from the historical cohort with ARDS unrelated to COVID-19. Among patients who underwent CTPA, patients with pulmonary embolism were compared to those with no pulmonary embolism. Then, the variables associated with pulmonary embolism were assessed by means of multivariable logistic regression analyses. Given the fact that the estimated number of events was low, only two variables could be entered in the maximal model. A two-tailed $\mathrm{P}$ value of less than 0.05 was considered as indicating statistical significance. We used SAS software, version 9.4 (SAS Institute), for all the analyses.

\section{Results}

\section{Study participants}

Over the pandemic period, 50 patients were admitted to our ICUs for acute respiratory failure with confirmed COVID-19. Out of them, 42 patients required intubation and met the criteria for ARDS. CTPA was performed in 29 patients (69\%), and repeated twice in 7 patients (17\%).

In the historical 3-year cohort, 199 patients were admitted to our ICU for ARDS. After excluding the 43 patients with extra-pulmonary ARDS, 156 patients with pulmonary ARDS unrelated to COVID-19 were retained in the analysis including 49 patients (31\%) with ARDS related to viral pneumonia. CTPA was performed in 51 patients (33\%), and repeated twice in 5 patients (3\%).

Patients with ARDS related to COVID-19 had a lower severity score at admission, less comorbidity except for a higher proportion of patients with hypertension, less severe hypoxemia within the first 48 hours of ARDS, and had lower mortality than those with ARDS unrelated to COVID-19 (Table 1).

\section{Primary outcome}

Incidence of pulmonary embolism was significantly higher in patients with ARDS related to COVID-19 than in those with ARDS unrelated to COVID-19: 40\% (17/42) vs. 3\%
(5/156), $\mathrm{P}=0.001$.

The proportion of patients with pulmonary embolism among those who had CTPA was also significantly higher in patients with ARDS related to COVID-19 than in the others: 59\% (17/29) vs. 10\% (5/51), $\mathrm{P}=0.0001$ (Table 1 and Figure 1). Patients with ARDS related to COVID-19 had lobar pulmonary embolism in $5 / 17$ cases (29\%), segmental embolism in $10(59 \%)$ and sub-segmental embolism in 2 (12\%). Eight patients (47\%) had bilateral pulmonary embolism.

\section{Secondary outcome}

Among the 80 patients having undergone CTPA, 22 patients (28\%) had pulmonary embolism including 17 patients (77\%) with ARDS related to COVID-19 and 5 (23\%) unrelated to COVID-19 (Table 2). The incidence of pulmonary embolism was significantly higher in ARDS related to COVID-19 than in the others: 59\% (17/29) vs. 10\% (5/51), $\mathrm{P}<0.0001$. In patients who had pulmonary embolism, CTPA was performed later after intubation as compared to patients with no pulmonary embolism: 8 [6-12] vs. 2 [1-8] days, $\mathrm{P}=0.0015$.

After multivariable logistic regression, ARDS related to COVID-19 remained independently associated with pulmonary embolism, even after adjustment on the duration between ICU admission and CTPA with an odds ratio of 13.0 (95\% confidence interval, 4.0 to 41.7$)$.

\section{Discussion}

In this single-center study, patients with ARDS related to COVID-19 had markedly higher incidence of pulmonary embolism than those with ARDS unrelated to COVID-19. Incidence of pulmonary embolism was $40 \%$ among all patients with ARDS related to COVID-19 and 59\% among those who had CTPA, the actual incidence being between these 2 rates.

The proportion of patients with ARDS related to COVID-19 and confirmed diagnosis of pulmonary embolism (40\%) was markedly higher than that reported in the literature, ranging from $17 \%$ to $24 \%$ among patients with respiratory failure (6-10). However, in the subgroup of ICU patients, one of these studies reported pulmonary embolism in $50 \%$ of patients who had CTPA, which was close to the $59 \%$ we have reported (8). Our study included only ARDS patients under invasive mechanical ventilation at the time of CTPA that was performed late in the course 
Table 1 Comparison of patients with acute respiratory distress syndrome (ARDS) related to COVID-19 and those with pulmonary ARDS unrelated to COVID-19

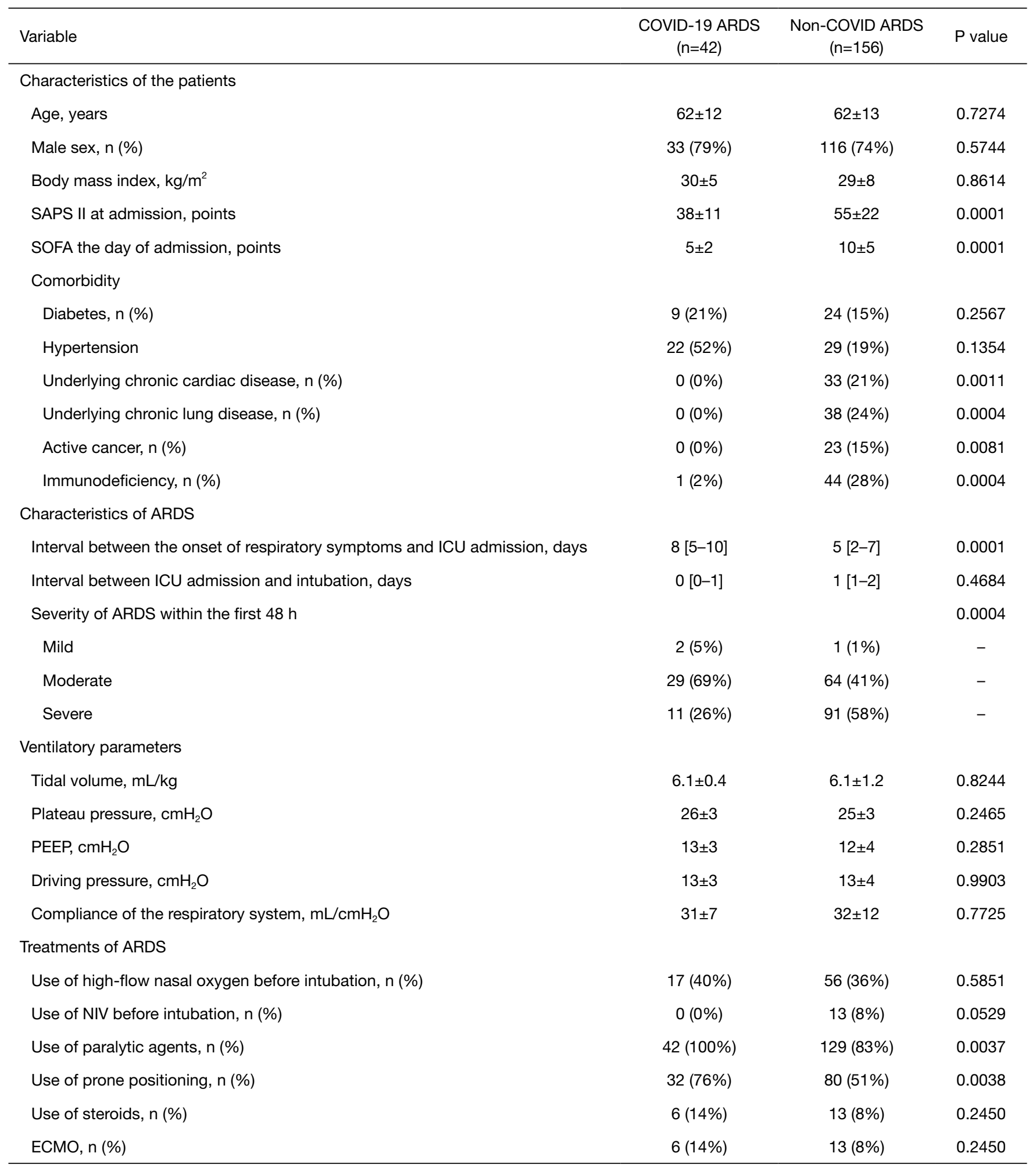

Table 1 (continued) 
Table 1 (continued)

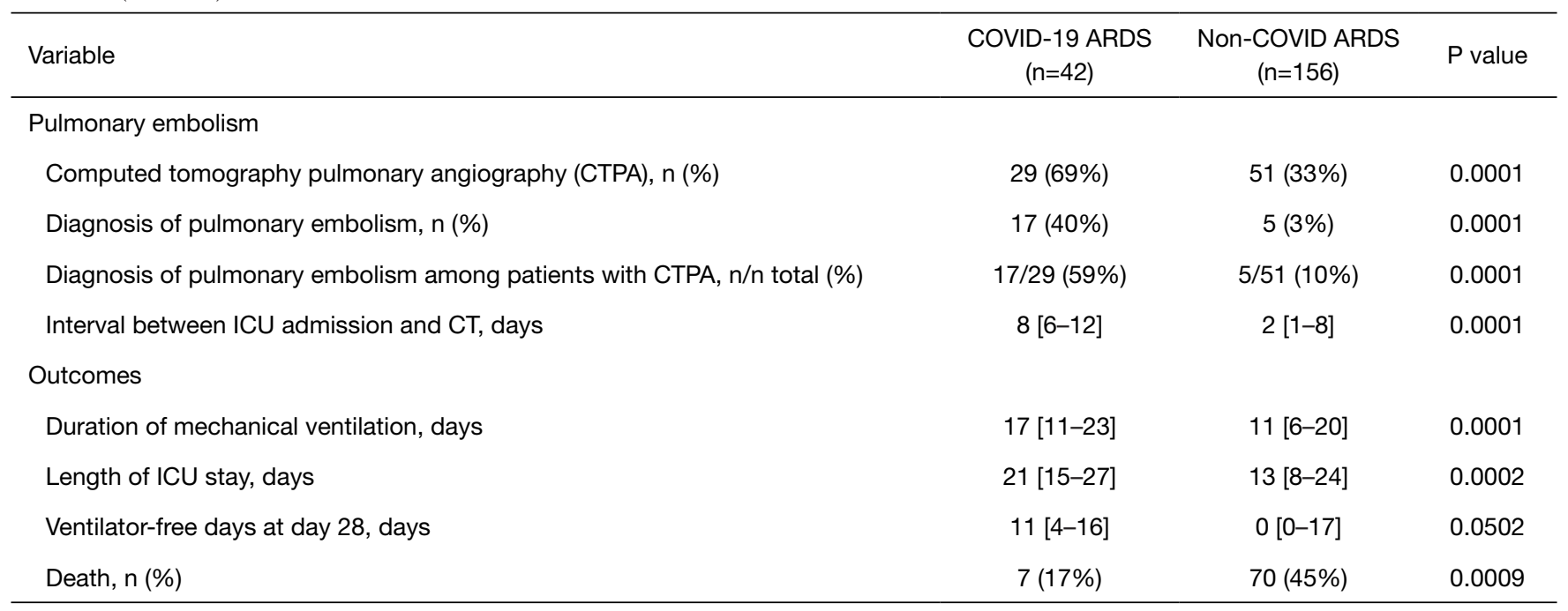

Continuous variables are given in mean \pm standard deviation and compared between the 2 groups using the Student's $t$-test, or given in median $\left[25^{\text {th }}-75^{\text {th }}\right.$ percentiles] and compared using a Mann-Whitney test according to their distribution, and categorical variables are given in number and percentage and were compared by means of the $\chi^{2}$ test. ARDS, acute respiratory distress syndrome; SAPS, simplified acute physiological score; SOFA, sepsis organ failure assessment; ICU, intensive care unit; NIV, non-invasive ventilation; CTPA, computed tomography pulmonary angiography; ECMO, extra-corporeal membrane oxygenation.

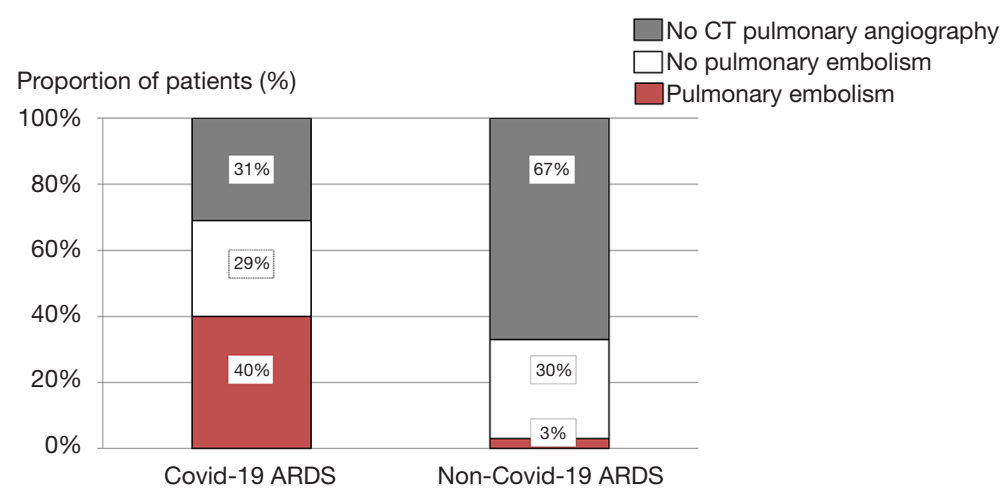

Figure 1 Bars showing the proportion of patients who had no computed tomography pulmonary angiography (CTPA) under mechanical ventilation (grey bars), those who had no pulmonary embolism on CTPA (white bars), and those who had confirmed diagnosis of pulmonary embolism on CTPA (red bars) in patients with ARDS related to COVID-19 and those with ARDS of non-COVID origin. The proportion of patients with pulmonary embolism was higher in COVID-19 ARDS than in those with ARDS of other origin (40\% vs. $3 \%$, $\mathrm{P}=0.0001)$. Although patients with COVID-19 ARDS had more frequently a CTPA than those with ARDS of other origin, the proportion of patients with confirmed diagnosis of pulmonary embolism among all patients who had CTPA was higher in COVID-19 ARDS than in the others (59\% vs. $10 \%, \mathrm{P}<0.0001)$. ARDS, acute respiratory distress syndrome.

of ARDS (8 days after ICU admission in median), and at times repeated if needed. In previous studies, CTPA was not systematic and was performed earlier which could explain why a number of pulmonary embolisms may have been overlooked.
To our knowledge, this is the first time that the incidence of pulmonary embolism was compared between ARDS related to COVID-19 and patients with pulmonary ARDS of other origin. Indeed, although several previous studies have compared the incidence of pulmonary embolism 
Table 2 Comparison of patients with acute respiratory distress syndrome (ARDS) and pulmonary embolism and those with no pulmonary embolism among all patients who had computed tomography pulmonary angiography (CTPA) after intubation

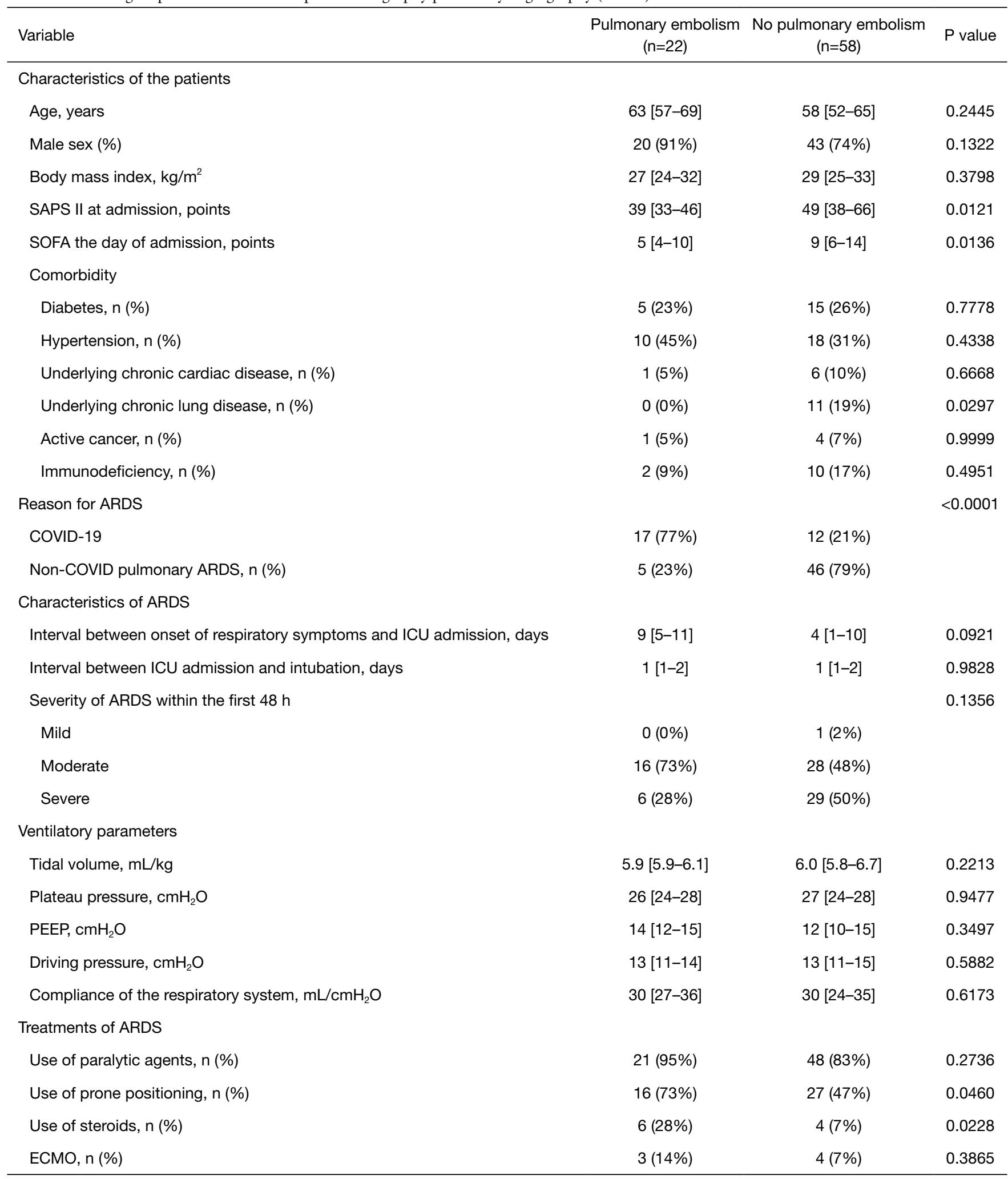

Table 2 (continued) 
Table 2 (continued)

\begin{tabular}{|c|c|c|c|}
\hline Variable & $\begin{array}{l}\text { Pulmonary embolism } \\
\qquad(\mathrm{n}=22)\end{array}$ & $\begin{array}{l}\text { No pulmonary embolism } \\
\qquad(n=58)\end{array}$ & $P$ value \\
\hline \multicolumn{4}{|l|}{ Timing of CTPA } \\
\hline \multicolumn{4}{|l|}{ Outcomes } \\
\hline Duration of mechanical ventilation, days & 18 [24-29] & 12 [8-23] & 0.0220 \\
\hline Ventilator-free days at day 28 , days & $10[0-13]$ & $5[0-17]$ & 0.6783 \\
\hline Death (\%) & $6(27 \%)$ & $17(29 \%)$ & 0.9999 \\
\hline
\end{tabular}

Continuous variables are given in median $\left[25^{\text {th }}-75^{\text {th }}\right.$ percentiles] and compared using a non-parametric Mann-Whitney test. Categorical variables are given in number and percentage and were compared by means of the non-parametric Fisher exact test or the $\chi^{2}$ test. ARDS, acute respiratory distress syndrome; SAPS, simplified acute physiological score; SOFA, sepsis organ failure assessment; ICU, intensive care unit; CTPA, computed tomography pulmonary angiography; ECMO, extra-corporeal membrane oxygenation.

between patients with ARDS related to COVID-19 and historical cohorts of ICU patients $(6,7)$, they encompassed either non-selected ICU patients (6) or patients with septic shock (7). Helms and colleagues reported an incidence of pulmonary embolism of $17 \%$ in patients with ARDS related to COVID-19 versus only $1 \%$ in a historical cohort of ARDS of other origin (7). However, patients with ARDS unrelated to COVID-19 were identified from a historical cohort including patients with septic shock and probably patients with extra-pulmonary ARDS. By contrast, our historical cohort included all consecutive patients with pulmonary ARDS.

\section{Limitations}

The main limitation of this study is the retrospective nature of the analysis. In the historical cohort, only one-third of patients with pulmonary ARDS unrelated to COVID-19 underwent CTPA, and it was performed much earlier after ICU admission than in patients with ARDS related to COVID-19. Consequently, the actual incidence of pulmonary embolism in patients with ARDS unrelated to COVID-19 could be markedly underestimated. To date, no study has prospectively assessed the incidence of pulmonary embolism in ARDS and we cannot exclude the eventuality that is necessary to perform CTPA later in the course of ARDS. Although we prospectively planned systematic CTPA to confirm or rule out pulmonary embolism after around one week of mechanical ventilation in all patients with ARDS related to COVID-19, CTPA was nonetheless performed in only $69 \%$ of patients, either because they were extubated earlier or because they were too severe to undergo scanner.

Another limitation is that we did not perform routine ultrasound examination of the lower limb veins and consequently we cannot hypothesize the mechanism of pulmonary embolism. Whereas the incidence of deep venous thrombosis may also be particularly high in patients with COVID-19 reaching approximately 40 to $50 \%$ of cases $(5,13,14)$, histological findings suggest that pulmonary embolism could be due to thrombosis of small pulmonary arteries rather than by thromboembolism (4).

Lastly, patients with ARDS related to COVID-19 had surprisingly few underlying chronic diseases, especially few chronic cardiac or lung diseases, and few cancers whereas these are strong risk factors for pulmonary embolism. The explanation is unclear but could in part be due to strict lockdown of patients at risk for COVID-19. Regardless the reason, this is in keeping with the recent French cohort including more than 4000 patients with COVID-19 in ICUs and reporting that only $7 \%$ of patients had underlying immunodeficiency (15).

In conclusion, pulmonary embolism was particularly frequent in patients with ARDS related to COVID-19, thereby suggesting that CTPA should be systematically performed during their ICU stay.

\section{Acknowledgments}

We thank Jeffrey Arsham (CHU de Poitiers, Poitiers, 
France) for reviewing and editing the original Englishlanguage manuscript.

Funding: None.

\section{Footnote}

Reporting Checklist: The authors have completed the STROBE reporting checklist. Available at http://dx.doi. org/10.21037/atm-20-6796

Data Sharing Statement: Available at http://dx.doi. org/10.21037/atm-20-6796

Peer Review File: Available at http://dx.doi.org/10.21037/ atm-20-6796

Conflicts of Interest: All authors have completed the ICMJE uniform disclosure form (available at http://dx.doi. org/10.21037/atm-20-6796). Dr. RC reports grants from French Intensive Care Society, grants from European Respiratory Society, grants from Fondation Poitiers Université, grants from Ecole doctorale Biosanté, outside the submitted work. Dr. JPF reports personal fees and non-financial support from Fisher and Paykel Healthcare, personal fees from SOS oxygène, grants from French ministry of health, outside the submitted work. Dr. AWT reports personal fees and non-financial support from Fisher \& Paykel, personal fees from Maquet-Getinge, personal fees from GE Healthcare, personal fees from Covidien, outside the submitted work. The other authors have no conflicts of interest to declare.

Ethical Statement: The authors are accountable for all aspects of the work in ensuring that questions related to the accuracy or integrity of any part of the work are appropriately investigated and resolved. The study was conducted in accordance with the Declaration of Helsinki (as revised in 2013), and was approved by the local Ethics committee of Poitiers (registration number: CHU86RECH-R2020-05-09). Given its observational nature, informed consent was waived.

Open Access Statement: This is an Open Access article distributed in accordance with the Creative Commons Attribution-NonCommercial-NoDerivs 4.0 International License (CC BY-NC-ND 4.0), which permits the noncommercial replication and distribution of the article with the strict proviso that no changes or edits are made and the original work is properly cited (including links to both the formal publication through the relevant DOI and the license). See: https://creativecommons.org/licenses/by-nc-nd/4.0/.

\section{References}

1. Wu C, Chen X, Cai Y, et al. Risk Factors Associated With Acute Respiratory Distress Syndrome and Death in Patients With Coronavirus Disease 2019 Pneumonia in Wuhan, China. JAMA Intern Med 2020;180:934-43.

2. Grasselli G, Zangrillo A, Zanella A, et al. Baseline Characteristics and Outcomes of 1591 Patients Infected With SARS-CoV-2 Admitted to ICUs of the Lombardy Region, Italy. JAMA 2020;323:1574-81.

3. Thille AW, Esteban A, Fernandez-Segoviano P, et al. Chronology of histological lesions in acute respiratory distress syndrome with diffuse alveolar damage: a prospective cohort study of clinical autopsies. Lancet Respir Med 2013;1:395-401.

4. Lax SF, Skok K, Zechner P, et al. Pulmonary Arterial Thrombosis in COVID-19 With Fatal Outcome: Results From a Prospective, Single-Center, Clinicopathologic Case Series. Ann Intern Med 2020;173:350-61.

5. Wichmann D, Sperhake JP, Lütgehetmann $M$, et al. Autopsy Findings and Venous Thromboembolism in Patients With COVID-19: A Prospective Cohort Study. Ann Intern Med 2020;173:268-77.

6. Poissy J, Goutay J, Caplan M, et al. Pulmonary Embolism in Patients With COVID-19: Awareness of an Increased Prevalence. Circulation 2020;142:184-6.

7. Helms J, Tacquard C, Severac F, et al. High risk of thrombosis in patients with severe SARS-CoV-2 infection: a multicenter prospective cohort study. Intensive Care Med 2020;46:1089-98.

8. Bompard F, Monnier H, Saab I, et al. Pulmonary embolism in patients with COVID-19 pneumonia. Eur Respir J 2020;56:2001365.

9. Gervaise A, Bouzad C, Peroux E, et al. Acute pulmonary embolism in non-hospitalized COVID-19 patients referred to CTPA by emergency department. Eur Radiol 2020;30:6170-7.

10. Sakr Y, Giovini M, Leone M, et al. Pulmonary embolism in patients with coronavirus disease-2019 (COVID-19) pneumonia: a narrative review. Ann Intensive Care 2020;10:124.

11. Bellani G, Laffey JG, Pham T, et al. Epidemiology, Patterns of Care, and Mortality for Patients With Acute Respiratory Distress Syndrome in Intensive Care Units in 
50 Countries. JAMA 2016;315:788-800.

12. Pelosi P, D'Onofrio D, Chiumello D, et al. Pulmonary and extrapulmonary acute respiratory distress syndrome are different. Eur Respir J Suppl 2003;42:48s-56s.

13. Voicu S, Bonnin P, Stépanian A, et al. High Prevalence of Deep Vein Thrombosis in Mechanically Ventilated COVID-19 Patients. J Am Coll Cardiol 2020;76:480-2.

Cite this article as: de Roubin V, Reynaud F, Coudroy R, Rodriguez M, Monseau G, Joly F, Bardin J, Boissier F, Chatellier D, Veinstein A, Robert R, Frat JP, Thille AW. High risk of pulmonary embolism in acute respiratory distress syndrome related to COVID-19: an observational controlledcohort study. Ann Transl Med 2021;9(8):630. doi: 10.21037/atm20-6796
14. Edler C, Schröder AS, Aepfelbacher M, et al. Dying with SARS-CoV-2 infection-an autopsy study of the first consecutive 80 cases in Hamburg, Germany. Int J Legal Med 2020;134:1275-84.

15. Clinical characteristics and day- 90 outcomes of 4244 critically ill adults with COVID-19: a prospective cohort study. Intensive Care Med 2021;47:60-73. 